\title{
Correlation Between IgA Tissue Transglutaminase Antibody Ratio and Histological Finding in Celiac Disease
}

\author{
${ }^{*}$ Maria G. Alessio, ${ }^{\dagger}$ Elio Tonutti, ${ }^{\ddagger}$ Ignazio Brusca, ${ }^{\S}$ Antonella Radice, $"$ Lisa Licini, \\ "Aurelio Sonzogni, "Ada Florena, ${ }^{\#}$ Eugenio Schiaffino, ${ }^{* *}$ Wally Marus, \\ ${ }^{* *}$ Sandro Sulfaro, and ${ }^{\dagger \dagger}$ Danilo Villalta, for the Study Group on Autoimmune \\ Diseases of the Italian Society of Laboratory Medicine
}

\begin{abstract}
Objectives: Positivity of both immumoglobulin A anti-tissue transglutaminase (TTG) and anti-endomysium antibodies (EMA) has a positive predictive value of nearly $100 \%$ for celiac disease (CD). The objective of the present study was to evaluate whether patients of any age, with high pretest probability of $\mathrm{CD}$ and high titre of anti-TTG and EMA positivity, have a high probability of intestinal damage and may not require the biopsy for final diagnosis.

Methods: A retrospective analysis of 412 consecutively referred patients, age range 10 months to 72 years, who underwent small-bowel biopsy for suspicion of CD and positivity to both anti-TTG and EMA, was performed at 4 Italian centers. Biopsies were evaluated independently by 2 pathologists using Marsh modified classification; in cases of dissimilar results, a third pathologist examined the biopsy. The final histological finding diagnosis was expressed as the prevalent or highest score assigned by the pathologist board.

Results: Three hundred ninety-six patients (96.1\%) had histological findings consistent with $\mathrm{CD}$ (grade 2 and $3 \mathrm{a}, 3 \mathrm{~b}$, or $3 \mathrm{c}$ of modified Marsh classification). An anti-TTG ratio $\geq 7$ was able to identify with the 3 assays used (Celikey, anti-TTG immumoglobulin A, EuTTG) all of the patients with significant mucosal damage (Marsh $\geq 2$ ) independent of age and sex; specificity and positive predictive value were $100 \%$. An anti-TTG ratio $>20$ was more specific $(99.8 \%)$ for identification of patients with villous atrophy (Marsh $3 \mathrm{a}, \mathrm{b}$, or c).

Conclusions: Patients with positivity of anti-TTG $\geq 7$-fold cutoff, confirmed by positivity to EMA, have a high-degree probability of duodenal damage. In selected conditions, a duodenal biopsy may be avoided and a confirmed
\end{abstract}

Received March 1, 2011; accepted December 16, 2011.

From the *Department of Laboratory Medicine, Biochemistry Laboratory, Riuniti Hospital, Bergamo, the †Immunopathology and Allergology Unit, S. Maria della Misericordia Hospital, Udine, the $\ddagger$ Department of Clinical Pathology, Buccheri La Ferla Hospital, Palermo, the §Microbiology and Virology Laboratory, San Carlo Hospital, Milan, the ||Department of Laboratory Medicine, Pathological Anatomy, Riuniti Hospital, Bergamo, the ${ }^{\top}$ Department of Human Pathology, University of Palermo, Palermo, \#Pathological Anatomy and Histology, San Carlo Hospital, Milan, **Pathological Anatomy, Santa Maria degli Angeli Hospital, and the ††Departement of Laboratory Medicine, Allergy and Clinical Immunology, S. Maria degli Angeli Hospital, Pordenone, Italy.

Address correspondence and reprint requests to Maria G. Alessio, Largo Barozzi, 1 I-24123 Bergamo, Italy (e-mail: mgalessio@ospedaliriuniti. bergamo.it).

The authors report no conflicts of interest.

Copyright $(1) 2012$ by European Society for Pediatric Gastroenterology, Hepatology, and Nutrition and North American Society for Pediatric Gastroenterology, Hepatology, and Nutrition

DOI: $10.1097 / \mathrm{MPG} .0 \mathrm{~b} 013 \mathrm{e} 3182470249$ greatly positive anti-TTG result could be the basis to prescribe a gluten-free diet.

Key Words: anti-TTG ratio, celiac disease, mucosal damage

(JPGN 2012;55: 44-49)

C eliac disease (CD) is an immune-mediated enteropathy caused by a permanent sensitivity to gluten in genetically susceptible individuals (1). First considered a rare malabsorption syndrome of childhood, $\mathrm{CD}$ is now recognized as a common condition that may be diagnosed at any age and that affects many organs and systems.

Clinical manifestations of CD vary greatly according to the extension and duration of disease, and to the age of the patient (2). Diagnosis of CD is based on a combination of clinical findings, positivity of serological tests, and histological demonstration of duodenal mucosal damage.

The anti-tissue transglutaminase (TTG) antibody assay is actually identified as the best test to screen patients with suspected CD. Anti-TTG assays using recombinant antigen show a high sensitivity compared with anti-endomysium antibodies (EMA) and a good positive predictive value (PPV); although an international standard was not available, systematic reviews have concluded that anti-TTG assay is the test with a better diagnostic performance for identifying $C D$ in adults and in children older than 2 years $(3-6)$.

The use of the EMA assay in daily practice is limited by high costs and subjective interpretation with unacceptable variability among laboratories that perform this test (6); however, because of its high specificity it is actually proposed in diagnostic protocols to confirm a positive result of anti-TTG antibody assay. A positivity of both anti-TTG antibody assay and EMA has a sensitivity and a PVV for CD of almost $100 \%$ (7).

In the 1990s, the European Society of Pediatric Gastroenterology, Hepatology, and Nutrition proposed a revised CD diagnostic protocol. Based on these revised criteria, if the anamnesis, the clinical history, and the screening results are suggestive of the disease, a single intestinal biopsy followed by a favorable response to a gluten-free diet (GFD) is sufficient to confirm the diagnosis of $\mathrm{CD}(8)$. Although biopsy is actually considered the criterion standard for the diagnosis of $\mathrm{CD}$, it has many limitations. The possible patchy distribution of the intestinal mucosal damage, and the incorrect orientation of the biopsy for appropriate evaluation of the villous morphology, limits the value of the biopsy $(6,9,10)$.

Complete villous atrophy is typical of $\mathrm{CD}$, but it is now considered the extreme end of a continuous spectrum of tissue 
damage that can be detected during the acute phase of the disease. In fact, according to the Marsh classification modified by Oberhuber $(11,12)$ the presence of an inflammatory infiltrate of CD3 lymphocytes $>25$ of 100 enterocytes (Marsh 1) alone or associated with hyperplasia of the crypts (Marsh 2) can be related to $\mathrm{CD}$ in the presence of symptoms and serology suggestive of disease (13); however, there is poor agreement among different pathologists in evaluating the entity of intestinal damage, particularly when it is minimal (14). Given the high performance of the screening test available and the limits of the duodenal biopsy, some authors proposed a modification of the diagnostic algorithm for $\mathrm{CD}$, providing the possibility of not resorting to biopsy in presence of high levels of TTG (15-18).

The aim of the present study was to evaluate whether, in patients with high pretest probability of disease, the positivity of both anti-TTG and EMA is associated in a high percentage of cases with significant duodenal damage (Marsh $\geq 2$ ), also in the presence of a small level of anti-TTG antibodies and independent of the assay used; this finding would make unnecessary the use of biopsy in carefully selected cases.

\section{METHODS}

Four hundred twelve consecutively investigated patients (134 males and 278 females; 249 age 12 years or younger; age range 10 months -72 years; mean age $17.41+16.83$, median 10 years) selected during 2006-2007 at 4 Italian centers (Riuniti Hospital, Bergamo; Buccheri-La Ferla Hospital, Palermo; Santa Maria degli Angeli Hospital, Pordenone; and S. Maria della Misericordia Hospital, Udine) were included. The patients had to respond simultaneously to the following criteria: suggestive symptoms for CD and/or risk factors, positivity to both anti-TTG antibodies and EMA, availability of hematoxylin/eosin-stained histological specimens, and immunohistochemistry for CD3.

Twenty-five patients (6\% of total: 14 adults and 11 age 12 years and younger) did not have any symptoms and were investigated because of risk factors only. Comorbid conditions and symptoms of enrolled patients are shown in Table 1. The serological tests performed at the participating centers immediately before biopsy were considered. Patients with contradictory serological tests (12 cases, all with low anti-TTG antibody level and EMA negative) or with immunoglobulin A ( $\operatorname{Ig} \mathrm{A})$ deficiency and patients receiving GFD or suspected of having started the diet before the histological evaluation were not included. Overall, selected patients represent approximately $70 \%$ of those who yearly undergo a biopsy for suspected $\mathrm{CD}$ at the 4 centers.

\section{Serological Data}

Serum anti-TTG antibodies and EMA were measured by the commercial enzyme-linked immunosorbent assays and indirect immunofluorescence tests conducted routinely at each center. Serum anti-TTG antibodies were measured using assays with human recombinant TTG as antigen. In particular, the following assays were used in the study: Celikey, Phadia GmbH, Freiburg, Germany (PH, 3 centers, 330 patients); anti-TTG IgA, Orgentec Diagnostika GmbH, Mainz, Germany (OR, 2 centers, 42 patients); and EuTTG, Eurospital, Trieste, Italy (EU, 1 center, 40 patients). Two centers changed the kits during the study period. The results were expressed in units per milliliter. A decisional cutoff calculated at the $95 \%$ confidence interval by a receiver operating curve, as described by Van Meensel et al (19), was used for each assay; consequently, we considered all of the samples with values greater than the following cutoff to be positive: $\mathrm{PH} 2.64 \mathrm{U} / \mathrm{mL}$; EU $3.69 \mathrm{U} / \mathrm{mL}$, OR $9.73 \mathrm{U} / \mathrm{mL}$.

To compare results obtained with different kits, because of lack of an internationally accepted standard method, results were expressed also as anti-TTG ratio; that is, the ratio between the value obtained for the single patient and the decisional cutoff, as previously described (18).

EMA were detected on cryostat sections of monkey esophagus at a starting dilution of 1:10, and the results were expressed as positive or negative. The commercial indirect immunofluorescence used by the participating centers were manufactured by INOVA, San Diego, CA (2 centers, 351 patients); Euroimmun,

TABLE 1. Distribution of presenting symptoms and risk factors in adults and children*

\begin{tabular}{lcc}
\hline & Children, $\mathrm{n}=249(\%)$ & Adults, $\mathrm{n}=163(\%)$ \\
\hline Symptom & & \\
Abdominal (pain, diarrhea, distension) & $196(79)$ & $65(40)$ \\
Failure to thrive & $27(11)$ & 0 \\
Short stature only & $12(5)$ & 0 \\
Weight loss only & $15(6)$ & $8(5)$ \\
Iron deficiency anemia & $40(16)$ & $50(49)$ \\
Constipation & $7(3)$ & $5(3)$ \\
Vomiting & $10(4)$ & $2(1)$ \\
Unexplained elevation of & $6(2.4)$ & $3(28)$ \\
Folate deficiency & 0 & $7(4)$ \\
Osteoporosis & 0 & $5(3)$ \\
Delayed puberty & 0 & $15(9)$ \\
Risk factor & & $29(18)$ \\
Celiac disease in a first-degree relative & $30(12)$ & $5(3)$ \\
Autoimmune thyroid disease & $17(7)$ & $3(2)$ \\
Type 1 diabetes mellitus & $7(3)$ & $2(1)$ \\
Sjogren syndrome & 0 & 0 \\
Primary biliary cirrhosis & 0 & $5(2)$ \\
Down syndrome & &
\end{tabular}

\footnotetext{
* Younger than 12 years.
} 
Lubeck, Germany (1 center, 40 patients); and Eurospital, Trieste, Italy ( 1 center, 21 patients). All of the centers involved in the study participated in the United Kingdom External Quality Assessment Scheme and in the Italian Biomedical Research Centre External Quality Program for celiac serology.

\section{Histological Evaluation}

Histological slides, obtained from multiple endoscopic biopsies of the distal portion of duodenum collected by esophagogastroduodenoscopy (EGD) and evaluated by the pathologist at the participating centers during the first evaluation of the patients, were reviewed by a second blinded expert pathologist; the histological lesions were classified according to Oberhuber-modified Marsh classification (12). All of the biopsies were performed by forceps. For each case hematoxylin-eosin- and CD3-stained slides were available; CD3 staining was routinely performed in cases of suspected celiac disease.

A third pathologist was involved in revision if results were not concordant (ie, $\geq 1$ Marsh grade) and, if the specimen resulted in different diagnosis by the pathologist team, the prevalent score assigned by the pathologist board was considered for final diagnosis. A modified Marsh grade $\geq 2$ was considered suggestive of the presence of $\mathrm{CD}$.

\section{Statistical Analysis}

For statistical analysis, histological diagnoses were scored as Marsh 0, 1, and 2, whereas scores $>3$ (3a, 3b, 3c) were converted to an ordinal scale of 3, 4, and 5, respectively. Spearman rank correlation and logistic regression were used to evaluate the association between Marsh scores and anti-TTG ratio.

\section{RESULTS}

A total of 412 patients were included in the study. In 4 patients $(0.9 \%$ of total), the specimen was found inadequate for diagnosis, due to insufficient sample, and was excluded from the study. As far as the remaining 408 patients, 246 (66.4\%) were ages 12 years and younger; the ratio between girls $(n=275)$ and boys $(n=133)$ was 2.07 , representative of the distribution of CD in the general population (20). The ratio between each anti-TTG antibody value and the corresponding decisional cutoff ranged between 1.01 and $>38.26$.

The results of the histological findings on biopsy obtained by EGD are shown in Table 2. Sixty-six of 408 samples (16\%) received a different histological classification given by 2 pathologists. In particular, a different grade of villous atrophy (Marsh 3a, b, or c) was assigned to 42 samples $(10.2 \%)$ and the highest score was recorded; 24 of $408(5.8 \%)$ of the total specimens collected received a Marsh classification-based diagnosis with at least 1 grade of difference and underwent a review by a third pathologist. Regarding the classification received at the time of diagnosis, 12 were reallocated to a higher degree, 8 to a lower degree, and in 4 cases, the samples were considered inadequate; however, the degree of correlation between the pathologists was satisfactory.

Twelve of 408 samples $(2.9 \%)$ had histology poorly consistent with CD: 6 had normal villous morphology and 6 showed only an increase in intraepithelial lymphocytes (CD3 $>25 / 100$ enterocytes) without atrophy and were classified as Marsh type 1; anti-TTG antibodies of these 12 patients ranged between 5.11 and $16.6 \mathrm{U} / \mathrm{mL}$ and the anti-TTG ratio ranged between 1.04 and 6.29.

Of these 12 patients, 8 had a final diagnosis of CD: 4 patients were classified as having $\mathrm{CD}$ at 1 reference center on the basis of serological data, presence of human leukocyte antigen (HLA) of

\begin{tabular}{lc}
\hline TABLE 2. Histological evaluation in 408 selected patients \\
\hline Histology & No. patients (\% total) \\
\hline Marsh 0 & $6(1.5)$ \\
Marsh 1 & $6(1.5)$ \\
Marsh 2 & $12(2.9)$ \\
Marsh 3 & $384(94.1)$ \\
3a & $39(10.2)$ \\
3b & $123(32.0)$ \\
3c & $222(57.8)$ \\
Total & $408(100)$ \\
\hline
\end{tabular}

susceptibility, suggestive symptoms and their disappearance, and negativity of serology after GFD. They showed a suggestive biopsy (Marsh 2) at the first histological evaluation, but afterward were classified as Marsh 0 (3 patients) and Marsh 1 (1 patient) by the pathologist board. Another 4 patients, all with Marsh 1, had risk factors ( 2 with autoimmune thyroid disease, 1 with Down syndrome, and 1 with 2 first-degree relatives affected) and positivity of serological tests at low levels. Three of these patients underwent a second biopsy after 4 months, and Marsh 1 score has been confirmed; 1 (Down syndrome patient) received a final diagnosis based on increased levels of anti-TTG and anti-EMA and worsening of symptoms, but biopsy was not repeated. Finally, 4 patients, 1 with Marsh 1 classification and 3 with normal mucosa, were lost to follow-up.

Three hundred ninety-six of 408 specimens (97\%) had a histology compatible with $\mathrm{CD}$, with inflammatory infiltrate and hyperplasia of the crypts (Marsh 2, $\mathrm{n}=12,2.9 \%$ ) or varying degrees of villous atrophy (Marsh 3a, b, or c: $n=384,94.1 \%$ ). All of these patients had a final diagnosis of CD. The correlation between antiTTG ratio, histological findings, and final diagnosis of CD for the different assays used in the study is depicted in Table 3.

Independent of age, sex, and commercial kit used for determining anti-TTG antibodies, already at a value of ratio $>2$, $91.6 \%$ of patients showed a mucosal atrophy (Marsh 3a, b, c), which is greatly suggestive of $\mathrm{CD}$; this percentage increased to $98.7 \%$ when a ratio $>7$ was considered. A value of anti-TTG ratio $>20$ had almost absolute correspondence with atrophic lesions (99.4\%) because only 1 patient showed no villous atrophy (Marsh 2). When Marsh 2 type was also included as criteria for suggestive $\mathrm{CD}$, a ratio value $>7$ showed a $100 \%$ sensibility and PPV for significant mucosal damage above this value; no patient had Marsh 0 or 1 . The PPV of anti-TTG ratio for presence of significant lesions (Marsh $\geq 2$ ), mucosal atrophy (Marsh 3a, b, c), and presence of $\mathrm{CD}$ is shown in Table 4.

\section{DISCUSSION}

$\mathrm{CD}$ is now generally accepted as a much more complex disease than previously thought, and hence a multidisciplinary approach is often required for its correct diagnosis (21).

Although histology is considered the criterion standard for the diagnosis of $\mathrm{CD}$, its limitations have become more apparent with the availability of even more accurate tests (HLA, anti-TTG assay, EMA assay, anti-deamidated gliadin peptides assay, subepithelial deposits of IgA) that have improved the diagnostic accuracy and have emphasized the limits of histological examination (22).

In the initial phases of the disease, CD-related mucosal damage and atrophy may occur discontinuously (9); therefore, at least 4 endoscopic biopsies must be taken from different parts of the 


\begin{tabular}{|c|c|c|c|c|c|c|c|}
\hline \multirow[t]{3}{*}{$<2.0$} & $\mathrm{PH}$ & $2\left(2^{*}\right)$ & $1^{\ddagger}$ & 4 & 4 & 11 & 9 \\
\hline & OR & $1^{\dagger}$ & 0 & 1 & 2 & 4 & 4 \\
\hline & EU & 0 & 0 & 0 & 4 & 4 & 4 \\
\hline $2.0-7.00$ & PH & $3\left(1^{*}\right)\left(2^{\dagger}\right)$ & $5\left(1^{*}\right)\left(1^{\dagger}\right)\left(3^{\ddagger}\right)$ & 1 & 44 & 53 & 51 \\
\hline \multirow[t]{3}{*}{$7.00-20.00$} & $\mathrm{PH}$ & 0 & 0 & 3 & 87 & 90 & 90 \\
\hline & OR & 0 & 0 & 1 & 30 & 31 & 31 \\
\hline & EU & 0 & 0 & 0 & 1 & 1 & 1 \\
\hline \multirow[t]{2}{*}{$>20$} & $\mathrm{PH}$ & 0 & 0 & 1 & 173 & 174 & 174 \\
\hline & OR & 0 & 0 & 0 & 0 & 0 & 0 \\
\hline
\end{tabular}

$\mathrm{CD}=$ celiac disease $; \mathrm{EU}=$ Eurospital; $\mathrm{OR}=$ Orgentec; $\mathrm{PH}=$ Phadia $; \mathrm{TTG}=$ anti-tissue transglutaminase antibodies.

* Patients with final diagnosis unavailable.

${ }^{\dagger}$ Patients who received diagnosis of CD at 1 reference center, based on positivity of serological tests, symptoms, and suggestive biopsy at the first histological evaluation (Marsh 2/3) but classified as Marsh 0 or Marsh 1 by the pathologist board.

${ }^{\ddagger}$ Patients with later diagnosis.

duodenal mucosa (23). The samples must be properly positioned, oriented, and sectioned. It is common knowledge that an incorrect preparation of the sample can simulate both a false shortness of the villi and an increased count of lymphocytes in epithelium and lamina propria, with an outcome of a false diagnosis of $\mathrm{CD}$ $(10,24,25)$. In some cases, especially in extremely young children, the amount of material collected may be insufficient to reach a definitive diagnosis; a European multicenter study showed that $>10 \%$ of histological specimens collected for suspected CD were insufficient for diagnosis (6). Another limiting factor of histological examination is the interobserver variability and the poor reproducibility among pathologists. Corazza et al (14) found that the degree of correlation among pathologists with similar experience did not exceed $75 \%$, and was even lower, around $25 \%$, for the initial grade of lesions (modified Marsh types 1 and 2), which may give rise to doubt or diagnostic errors. Finally, in early life, EGD is generally performed under anesthesia, with increased risk and reduced compliance of parents to subject the child to an invasive examination.

Many studies have tried to establish a correlation between autoantibody levels and extent of mucosal damage, with the aim of limiting the number of endoscopies required for the diagnosis of CD. A European multicenter study by the International Working Group on Eu-TG showed that in children, more severe degrees of intestinal damage correspond to higher levels of antiTTG antibodies (26).
Hansson et al (27) demonstrated a correlation between antiTTG antibody levels and mucosal damage in a cohort of 57 children with a final diagnosis of $\mathrm{CD}$; the same results were obtained by Tursi et al (28) in adult subjects using a guinea pig anti-TTG assay; they also suggested a possible role for anti-TTG antibodies in predicting mucosal damage, independent of the source of the TTG.

Scoglio et al (29) first showed the high pretest predictive value of anti-TTG antibodies and EMA, assuming the avoidance of intestinal biopsy to confirm a clinical suspicion of $\mathrm{CD}$, but other authors proposed finding a value of anti-TTG antibodies able to identify a mucosal injury suggestive of $\mathrm{CD}$, and, consequently, to eliminate the need for EGD in some selected cases.

Diamanti et al (16) suggested that in patients with symptoms, there was a strong correlation between anti-TTG antibody levels and degree of mucosal injury, and demonstrated that a anti-TTG cutoff value $\geq 20 \mathrm{U} / \mathrm{mL}$ ( 5 times the cutoff) was suitable for predicting mucosal atrophy.

In a retrospective evaluation on 103 children who had undergone EGD following positive anti-TTG antibody assay, Barker et al (15) showed that 48 of 49 patients with anti-TTG $>100 \mathrm{U} / \mathrm{mL}$ ( 5 times the cutoff) had histological findings of modified Marsh $\geq 2$, which confirms the suspected diagnosis of $\mathrm{CD}$. Sensibility and specificity of anti-TTG $>100 \mathrm{U} / \mathrm{mL}$ were $98 \%$ and $97.2 \%$, respectively; these results were subsequently confirmed by other authors $(17,18)$. Hill et al $(18)$ proposed the use of the ratio between the value of anti-TTG antibodies of the patient and the decisional

TABLE 4. PPV of TTG ratio for suggestive histological lesions (Marsh $\geq 2$ ) and CD

\begin{tabular}{lcccrrr}
\hline TTG ratio & No. patients & Marsh 3 (a, b, c) only & PPV & Marsh $\geq 2$ & PPV & CD \\
\hline$<2.0$ & 19 & 10 & 52.6 & 15 & 78.9 & 17 \\
$>2.0$ & 389 & 374 & 96.1 & 381 & 97.9 & 386 \\
$>5.0$ & 333 & 326 & 97.9 & 329 & 98.8 & 331 \\
$>10.0$ & 263 & 259 & 98.5 & 263 & 100 & 263 \\
$>20.0$ & 174 & 173 & 99.4 & 174 & 100 \\
\hline
\end{tabular}

$\mathrm{CD}=$ celiac disease $; \mathrm{PPV}=$ positive predictive value; $\mathrm{TTG}=$ anti-tissue transglutaminase antibodies. 
cutoff, instead of the absolute value, to compare results obtained with different kits in the absence of an international standard. The study showed that in 148 adults selected on the basis of positivity of both anti-TTG antibodies and EMA and presence of suggestive symptoms or risk factors, a ratio $>10$ was able to identify the subjects with intestinal lesions corresponding to a Marsh $\geq 2$ and therefore suggestive for the presence of CD. All of the mentioned authors concluded that there was the possibility not to perform EGD in patients with high pretest probability of CD and high anti-TTG antibody levels.

Our study shares this goal, showing that for the 3 different anti-TTG assays evaluated, an anti-TTG ratio $>7$ is high enough to identify all of the subjects with Marsh $\geq 2$, even if a limitation of the present study remains the lower number of subjects tested with OR and EU assays in comparison with the subjects tested with $\mathrm{PH}$ assay.

To our knowledge, this is the first multicenter study conducted in 4 main Italian hospitals, located in different regions and representative of the entire Italian health system. Possible limitations of the study should be due to the fact that patients were selected by expert clinicians and laboratory examinations performed by expert pathologists, so that the results of the study could not be extended or reproduced in a less expert setting; however, the number of patients is more than that in the previous studies and the present study evaluated a mixed populationpediatric and adult. Another limitation could be the retrospective data collection, and as a consequence the exclusion of some patients for incomplete data or sera collected at different time points than biopsies.

We selected only patients with high pretest probability of $\mathrm{CD}$ (suggestive symptoms, risk factors, comorbid conditions) and with positivity of anti-TTG antibodies and EMA. Some clinical conditions, such as liver disease, rheumatoid arthritis, and inflammatory bowel disease, can cause a falsely positive anti-TTG result $(30,31)$, but when both anti-TTG and EMA are positive in a patient with suggestive symptoms and/or risk factors, the probability that the patient had CD is extremely high, almost $100 \%$ (7). Furthermore, most Italian laboratories use the diagnostic protocol proposed by the Autoimmunity and Allergology Study Group of the Italian Society of Laboratory Medicine, which provides an initial screening with a highly sensitive test (anti-TTG) confirmed, if positive, by EMA (32).

The high specificity of EMA for CD was confirmed by the Finnish group of Maki and Kaukinen (33). They demonstrated that symptomatic children who are EMA positive without villous atrophy have celiac disorders; they benefit from early treatment with GFD, with disappearance of autoantibody and resolution of symptoms. The authors concluded that EMA positivity could be a sufficient criterion for $\mathrm{CD}$ diagnosis, despite normal mucosal structure. Similar results were obtained by the same authors in adults (34).

In our study, in addition to the pathologist of the recruitment center, the biopsy of every selected patient was examined by another gastrointestinally oriented pathologist, reducing the risk of errors and making more reliable the most critical aspect of the study, whose outcome is predominantly based on biopsy results.

The low number of unfit biopsies and the satisfactory agreement between the pathologist board—only 5.8\% samples with significant differences ( $\geq 1$ grade of Marsh classification) required a review by a third pathologist—can be explained by the large experience of the pathologists of participating centers and the higher percentage of biopsy with moderate to severe mucosal lesions (20). Biopsy remains a formidable but high-cost and time-consuming tool, not available in all of the institutions and requiring a pathologist confident in gastrointestinal pathology, particularly in cases with minimal duodenal mucosal lesions.
A possible limitation of our study is the small number of patients with a low degree of intestinal mucosal damage that is easily underestimated by a nonexperienced pathologist (Marsh 0 and 1), but it is due to the fact that the criteria of patient selection (positivity of serological tests and symptoms or risk factors) are associated with a high probability of finding significant mucosal damage. Furthermore, in many cases practitioners use caution in prescribing endoscopy in subjects with low levels of autoantibodies, mainly in infants, and prefer to wait and follow up with the patients.

As proposed by Hill et al (18), we expressed the results of anti-TTG as a ratio with the cutoff value calculated at the $95 \%$ confidence interval by a receiver operating curve (19) to compare results obtained at different centers with different commercial kits and instrumentation; our results are, however, similar to those of other groups. It is interesting to note that all of the authors who investigated the correlation between anti-TTG antibody levels (as ratio or absolute value) and degree of intestinal lesions reached the same conclusion: high levels of autoantibodies had strong sensitivity, specificity, and PPV for presence of intestinal damage consistent with $\mathrm{CD}$. In this regard, it appears suggestive because, in the works mentioned, the cutoff identified by the authors, when expressed as an anti-TTG antibody ratio, is surprisingly similar $(>5)$, regardless of the population selected and the type of test used at every center. The results and the number of patients evaluated appear sufficiently large and consistent to seriously consider modifying national and international guidelines (35).

Catassi and Fasano (36) suggested that the rigid and often complicated algorithms used to classify $\mathrm{CD}$ are unable to cover all of the faces of the disease, so they proposed to classify as CD all of the subjects with at least the presence of 4 criteria on 5 (presence of HLA-DQ2 and/or -DQ8; typical symptoms; high titre of serum celiac disease autoantibodies, celiac enteropathy at the small bowel biopsy and response to GFD); 3 criteria were considered sufficient if HLA was not performed. This proposal should be discussed and approved by the international scientific community, but it has the advantage of being extremely flexible and includes the possibility of excluding EGD only in selected patients evaluated at reference centers, with presence of all other criteria, including high level of autoantibodies and positivity of both anti-TTG and EMA. This approach could ensure the best compliance of patients and economic advantage for the sanitary system.

\section{CONCLUSIONS}

Our work shows that patients with suggestive symptoms and/ or risk factors for CD and positivity of both anti-TTG antibodies and EMA have a high likelihood of having duodenal damage and a final diagnosis of CD. Regardless of the age and sex of the subject and the type of commercial kits used in the present study, an anti-TTG antibody ratio $>7$ has $100 \%$ sensitivity, specificity, and PPV for the presence of histological lesions Marsh $\geq 2$ and diagnosis of CD. A ratio $>20$ had an almost absolute $(99.8 \%)$ correspondence with atrophic lesions (Marsh 3a, b, c) and 100\% PPV for CD.

The present study supports the findings of other authors, and confirms that in selected population, high levels of autoantibodies are correlated with a higher degree of mucosal damage and presence of $\mathrm{CD}$ in almost all of the cases.

We think that it is time to modify the guidelines for the diagnosis of CD and to adopt more flexible and simple criteria to facilitate more rapid diagnosis and treatment.

\section{REFERENCES}

1. Kagnoff MF. Overview and pathogenesis of celiac disease. Gastroenterology 2005;128:S10-8. 
2. Dewar DH, Ciclitira PJ. Clinical features and diagnosis of celiac disease. Gastroenterology 2005;128:S19-24.

3. Hill ID. What are the sensitivity and specificity of serological tests for celiac disease? Do sensitivity and specificity differ in different populations? Gastroenterology 2005;128:S25-32.

4. Rostom A, Dube C, Cranney A, et al. The diagnostic accuracy of serologic tests for celiac disease: a systematic review. Gastroenterology 2005;128:S38-46.

5. Hill PG, McMillan SA. Anti-tissue transglutaminase antibodies and their role in the investigation of coeliac disease. Ann Clin Biochem 2006;43:105-17.

6. Collin P, Kaukinen K, Vogelsang H, et al. Anti-endomysial and antihuman recombinant tissue transglutaminase antibodies in coeliac disease: a biopsy proven European multicenter study. Eur J Gastroenterol Hepatol 2005;17:85-91.

7. Hill ID, Dirks MH, Liptak GS, et al. Guideline for the Diagnosis and Treatment of Celiac Disease in Children: recomendations of the North American Society for Pediatric Gastroenterology, Hepatology and Nutrition. J Pediatr Gastroenterol Nutr 2005;40:1-19.

8. Walker-Smith JA, Scmerling DM. Revised criteria for diagnosis of coeliac disease. Report of Working Group of European Society of Paediatric Gastroenterology and Nutrition. Arch Dis Child 1990; 65:909-11.

9. Bonamico M, Mariani P, Thanasi E, et al. Patchy villous athrophy of the duodenum in childhood celiac disease. J Pediatr Gastroenterol Nutr 2004;38:204-7.

10. Perera DR, Weinstein WM, Rubin CE. Small intestinal biopsy. Hum Pathol 1975;6:157-217.

11. Marsh MN, Crowe PT. Morphology of the mucosal lesion in gluten sensitivity. Baillieres Clin Gastroenterol 1995;9:273-93.

12. Oberhuber G, Granditsch G, Vogelsang H. The histophatology of coeliac disease: time for a standardized report scheme for pathologists. Eur J Gastroenterol Hepatol 1999;11:1185-94.

13. Corazza GR, Villanacci V. Coeliac disease: some considerations on the histological diagnosis. J Clin Pathol 2005;58:573-4.

14. Corazza GR, Villanacci V, Zambelli C, et al. Comparison of the interobserver reproducibility with different histologic criteria used in celiac disease. Clin Gastroenterol Hepatol 2007;5:838-43.

15. Barker CC, Mitton C, Jevon G, et al. Can tissue transglutaminase antibody titers replace small bowel biopsy to diagnose celiac disease in selected pediatric populations? Pediatrics 2005;115:1341-6.

16. Diamanti A, Colistro F, Calce A, et al. Clinical value of immunoglobulin A antitransglutaminase assay in the diagnosis of celiac disease. Pediatrics 2006;118:1696-700.

17. Donaldson MR, Firth SD, Wimpee H, et al. Correlation of duodenal histology with tissue transglutaminase and endomysial antibody levels in pediatric celiac disease. Clin Gastroenterol Hepatol 2007;5:567-73.

18. Hill PG, Holmes KT. Coeliac disease: a biopsy is not always necessary for diagnosis. Alim Pharmacol Ther 2008;27:572-7.
19. Van Meensel B, Hiele M, Hoffman I, et al. Diagnostic accuracy of ten second generation (human) tissue transglutaminase antibody assays in celiac disease. Clin Chem 2004;50:2125-35.

20. Green PH, Jabri B. Celiac disease. Ann Rev Med 2006;57:207-21.

21. Villanacci V, Catassi C, Rostami K, et al. Coeliac disease: changing dogma on historical diagnosis. www.gastrohep.com. Accessed January $5,2010$.

22. Salmi TT, Collin P, Reunala T, et al. Diagnostic methods beyond conventional histology. Dig Liver Dis 2010;42:28-32.

23. Weir DC, Glickman JN, Roiff T, et al. Variability of histopathological changes in childhood enteropathies with special reference to intraepithelial lymphocytes. J Pediatr Gastroenterol Nutr 1982;1:525-53.

24. Rubin CE, Brandborg LL, Phelps PC, et al. Studies of celiac disease. I. The apparent identical and specific nature of the duodenal and proximal jejunal lesion in celiac disease and idiopathic sprue. Gastroenterology 1960;38:28-49.

25. Shidrawi RG, Przemioslo R, Davies DR, et al. Pitfalls in diagnosing coeliac disease. J Clin Pathol 1994;47:693-4.

26. Fabiani E, Catassi C. International Working Group on Eu-TG. The serum IgA class anti-transglutaminase antibodies in the diagnosis and follow up of celiac disease: results of an European multi-centre study. Eur J Gastroenterol Hepatol 2001;13:659-65.

27. Hansson T, Dahlbohm I, Rogberg S, et al. Recombinant human tissue transglutaminase for diagnosis and follow up of childhood celiac disease. Pediatr Res 2002;51:700-5.

28. Tursi A, Brandimarte G, Giorgetti GM. Prevalence of antitissue antitransglutaminase antibodies in different degrees of intestinal damage in celiac disease. J Clin Gastroenterol 2003;36:219-21.

29. Scoglio R, Di Pasquale G, Pagano G, et al. Is intestinal biopsy always needed for diagnosis of celiac disease? Am J Gastroenterol 2004, 98:1325-31.

30. Bizzaro N, Villalta D, Tonutti E, et al. IgA and IgG tissue transglutaminase antibody prevalence and clinical significance in connective tissue disease, inflammatory bowel disease and primary biliary cirrhosis. Dig Dis Sci 2003;48:2360-5.

31. Carroccio A, Di Prima L, Falci C, et al. Predictive value of serological tests in the diagnosis of celiac disease. Ann Ital Med Intern 2002; 17:102-7.

32. Tonutti E, Visentini D, Bizzaro N, et al. Linee guida per la diagnosi di laboratorio e istologica della malattia celiaca. RIMel/IJLaM 2005; 2:110-22.

33. Kurppa K, Ashorn M, IItanen S, et al. Celiac Disease without villous atrophy in children: a prospective study. J Pediatr 2010;157:373-80.

34. Kurppa K, Collin P, Viljamaa M, et al. Diagnosing mild enteropathy celiac disease: a randomized, controlled clinical study. Gastroenterology 2009;136:816-23.

35. Kaukinen K, Lindfors $\mathrm{K}$, Collin $\mathrm{P}$, et al. Review: celiac disease-a diagnostic and therapeutic challenge. Clin Chem Lab Med 2010; 48:1205-16.

36. Catassi C, Fasano A. Celiac disease diagnosis: simple rules are better than complicated algorithms. Am J Med 2010;123:691-3. 